\title{
Treatment of Refractory Lateral Epicondylitis of Humerus with Local Autologous Plasma Injection
}

\author{
Dr.Prosanta Roy ${ }^{1}$,Dr.P.Mondal ${ }^{2}$,Dr.A.Chakraborty ${ }^{3}$, \\ ${ }^{1}$ Assistant Professor,Nrs Medical College, Kolkata \\ ${ }^{2}$ Assistant Professor,Medical College, Kolkata \\ ${ }^{3}$ SR,Nrs Medical College,Kolkata
}

\begin{abstract}
:
Background: Elbow lateral epicondylitis is a common problem that usually resolves with conservative treatment modalities like local infiltration with steroids, ultrasonic therapy, and manipulation and finally operative procedures are tried. An alternative to surgical intervention is sought for when the conservative measures failed. The hypothesis of this study is to establish the role of local autologous plasma injection to bring alone biological cure in these cases.

Material and Methods: Forty patients with persisting pain for approximate six months, despite of other conservative mode of intervention were treated. The patients were injected with 2cc autologous plasma locally at the site of epicondylitis aseptically after preparation by centrifugation of $5 \mathrm{cc}$ blood. A post procedure physiotherapy program with active range of exercise was followed in every patient. Outcome was rated by post procedure visual analog pain scores (VAS) and Mayo elbow performance scores (MEPS). Informed consent had been obtained from the subjects.

Result: All patient in this study noted improvement in their VAS and MEPS. Minimal complication occurred in same patients. Mean VAS improved from 74.9 to start with to 41.5 at 4 weeks, 29.8 at 8 and 18.5 at 6 months follow up. Mean MEPS improved from 50.3 to start with to 71.8 at 4 weeks, 79.7 at 8 weeks and 84 at 6 months follow up. Conclusion: Treatment of patient with refractory lateral epicondylitis with local autologus plasma reduced pain significantly. Hence, local autologus plasma injection is a viable option to be considered before contemplating surgical intervention in refractory lateral epicondylitis of humerus.
\end{abstract}

Keyword: Refractory lateral epicondylitis, tennis elbow, tendinitis, tendinosis.

Study: Cohort study, level of Evidence 1.

\section{Introduction}

Lateral epicondylitis (tennis elbow) is a familiar term used to describe myriad symptoms along with activity-related pain around the lateral aspect of the elbow, with a common age of onset of 35 top 50 yrs and affecting around 4 adults per thousand annually. Activities requiring supination and pronation movments in near full elbow extended position leads to lateral epicondylitis of humerus.

Lateral epicondylitis is initiated by micro tear, most often, in the origin of the extensor crapi radialis brevis. Chronic overuse injuries following multiple microtraumatic events and cause disruption of the internal structure of the tendon and degeneration of the cells and matrix. These fail to mature into normal tendon, at times, leading to tendinosis ${ }^{2}$. The term tendinitis is used frequently to describe these condition, but, histopathological studies have shown that specimens of tendon obtained from areas of chronic overuse do not contain large number of macrophages, lymphocytes or neutrophils ${ }^{3,4}$. Histopathologically tendinosis have been found to be a population of fibroblasts, vascular hyperplasia, and disorganized collagen which is termed as angiofibroblastic hyperplasia ${ }^{5}$. Tendinitis on the contrary is characterized by the presence of an increased number of lymphocytes or neutrophils.

The cause of tendinosis to be painful is unclear and the cause of failure of collagen maturation is unknown. Tendons that are susceptible to injury are those that are involved in locomotion and ballistic performance, transmitting loads under elastic and eccentric conditions ${ }^{2,3,6}$ tendon running around a convex surface or the apex of a concavity, those crossing two joints, those with areas of scanty vascular supply, and those that are subjected to repetitive tension, are susceptible to overuse injury ${ }^{4,7,8,9,10}$.

Cyriax noted in his study that the origin of the extensor carpi radialis brevis was the primary site of this injury, and histopathological changes have been documented at this location ${ }^{4,10,11,12}$. Involvement of the origin of the extensor digitorum communis is also found in one-third of patients ${ }^{4,12,13}$. If it is assumed that tendinosis have basically the same pathogenesis, then the biologic methods of treatment of patients with tennis elbow can serve as a method for the treatment of pain in other regions in which tendinoses has been reported, like the patellar ligament, rotator cuff, flexer of the elbow, triceps, planter fascia and Achilles tendon.Plasma probably helps recruit bone marrow derived stem cell to the site of injection and plasma derived growth factors modulate the 
microvascular environment leading to healing process,further study into the mechanism of action of local autologous plasma is needed.

We chose to study lateral epicondylitis because it is clinically a very common entity and greatly hampers activity of daily living.our hypothesis is that local autologous plasma injection in treatment of cases of refractory lat epicondylitis of humerus promotes biological healing and yield better functional results.

\section{Methods}

The study was conducted in the Depeartment of Orthopaedics, Medical College and Hospitals, Kolkata on a prospective basis from February 2013 to July 2014. Out of the patients attending the out Patient Department of Orthopadics, Medical College and Hospitals,Kolkata and diagnosed as suffering from lateral epicondylitis of humerus, 40 patients were selected who fitted our criteria for the study. A written consent was obtained from all the patients. Inclusion criteria included adult patients(age group 20yr-60yr)suffering from lateral epicondylitis of humerus (after confirmation of diagonosis),pain persisting for atleast 6 months and failure of conservatine therapy including rest,activity modification,brasing, physiotherapy, steroid and nsaids. Exclusion criteria included pragnency,present of history of carpal tunnel syndrome,servical radiculopathy, significant intra-artiicular pathology,LCL Injury and systemic disorder.

Every patient was assessed and a detailed history of the patient was taken, noting the duration of pain, the mode and severity of injury, if any and physical examination. The case is again evaluated to conform the diaginesis by using the several tests for tennis elbow, such as Thomsen's Test, Mill's Maneuver and Cozen's Test

Anteroposterior and lateral projection of radiographs of the affected elbow were checked in all cases to exclude obvious pathology of trauma or cubital tunnel osteophytes. Colltral ligament injury and neuritis were excluded by clinical or imaging studies. Sonography was used for the evaluation of trendon and ligament condition.All the patients were evaluated by VAS and MEPS. The plasma for injection was prepared in the IHTM, Medical college Kolkata.

After proper counseling and following asepetic precaution about $5 \mathrm{cc}$ of blood was drawn in a syringe contacting $0.5 \mathrm{cc}$ of heparin from the patients contralateral forearm vein. The blood was centrifuged at 1300rbm for approxmently 20 to $25 \mathrm{mins}$ to generate the plasma. The duration from drawing of blood and injecting the plasma was 30 to 45 mins. The local area is then painted and draped. With $22 \mathrm{G}$ needle was advanced perpendicular to epicondyle till it touches the bone. Then the needle is slightly withdrawn by 1 to 2 millimeter and $2.5 \mathrm{ml}$ of plasma was injected into the area of maximum tenderness. The technique evolved in a single skin entry and 5 penetration of the tendon.

Immediately after the injection, the patient was kept in a supine position without moving the arm for 15 minutes, Patients was sent home with a compression bandage and instructions to limit their use of the arm for approximately 24 hours and use oral NSAIDS for 3 days for pain and local ice pack, if required. After 24 hours, patients were given a standardized stretching protocol to follow for 2 weeks. Patients were told to continue activities of normal daily living but to avoid any activities that were likely to aggravate symptoms. A formal strengthening program was initiated after this stretching. At 4 weeks after the procedure, patient were allowed to proceed with normal activities as tolerated. The patients were examined at 4 weeks, 8 weeks, and 6 months after the procedure. On every follow up visit, the patients were examined thoroughly.

\section{Result}

The patients ranged from 26 to 56 years (average 41.4 years). The most frequent age group affected was 30-39 yrs. They all underwent injection procedure and were followed up in this hospital on out patient basis for a period ranging from 6 to 14 months (average 8 months). 18 amongest the 40 patients were males and 22 among them were females. Sex distribution can be stated to be equal in incidence. Among the 40 patients, 21 were housewives, 9 were farmers, 9 were daily labourers and 4 were desktop workers. Amongst the 40 patients selected,in 28 the dominant upper limb was affected and in 12 the non-dominant side was affected.

Mayo elbow performance scoring system is the most widely used scoring system in cases of analysis of elbow pathology. It provides good analysis of the functional outcome of procedures done around the elbow.So we used the Mayo elbow performance score for analyzing our result as shown in the table.

The presence of pain was graded as none, mild, moderate and severe, 33 out of the total 40 cases $(82.5 \%)$ had moderate pain and 7 out of $40(17.5 \%)$ had severe pain at the pre procedure level. At 6 months follow up, 8 out of $40(20 \%)$ had none pain, 30 out of $40(75 \%)$ had mild pain, 2 out of 40 cases $(5 \%)$ had moderate pain. The function to comb hair improved from 10 out of 40 cases (97.5\%) at 6 months follow up, hygiene function improved from 19 cases $(47.5 \%)$ pre procedure to 38 cases(95\%) at 6 months follow up, dressing function improved from 1 case (2.5\%) pre procedure to 33 cases (82.5\%) at 6 months follow up, function improved from 2 cases (5\%) pre procedure to 23 cases $(57.5 \%)$ at 6 months follow up. Initially the patient selected for the study had a mean Mayo Elbow Score of 50.25, which at 4 weeks follow up showed a mean of 79.75 and at 6 months follow 
up improved to 84. Result interpretation based on MEP Score showed improvements as excellent in 7,good in 29, fair in 3 and poor in 1.

A $100 \mathrm{~mm}$ visual analog pain score (0-no pain, 100 worst pain possible) with colour coding is widely used to evaluate the subjective interpretation of pain. Initially the patient selected for the study had a mean VAS of 74.9 , which at 4 weeks follow up showed a mean of 41.5, 8 weeks follow up showed a mean of 29.8 and at 6 months follow up improved to 18.5 .

The changes in the mean values of the MEPS and the VAS from the pre procedure to each follow up level was assessed by ANOVA (Analysis of Variance). Based upon the results of the above mentioned statiscal analysis we concluded that the difference we obtained between the modalities of evaluation was statistically significant $(\mathrm{P}<0.0001)$. Pain (moderate) with associated functional difficulties persisted in $2(5 \%)$ cases. Severe pain was not present in any cases at 6 months follow up. No other cases had any other complications of infection, local skin condition changes, neuro-vascular deficit distally. 24 patients complained of increased pain following procedure which was relieved with analgesics and local ice pack application within 5 days.

\section{Discussion}

Lateral epicondylitis of humerus is a common problem usually controlled by conservative modalities. If conservative methods do not provide satisfactory remedy, other options are tried. A meta-analysis of physical therapy, recently, failed to provide evidence to establish it as a treatment modality providing long term relief ${ }^{14}$. Physical therapy, though, is also used in our study in the post procedure stretching and strengthening exercises. Corticosteroid injections have been used most popularly and extensively for treatment, but studies have raised certain doubts about their efficacy ${ }^{15,16}$. Superficial injection of corticosteroid injections may result in subcutaneous atrophy and sometimes leading to permanent ultra structural changes following intratendinous injection, as observed by Jobe and Ciccotti ${ }^{17}$. In our study this sort of complication did not occur as this is abiologic form of treatment. Extracorporial shock wave therapy was shown to be no better than placebo in a recent study ${ }^{18}$.in present day orthopaedics biological treatment modalities are gaining popularity.bone morphogenic protin and other growth factors have been studied in vitro and presently are being tested in vivo also ${ }^{19}$.the study by Edwards and calandrucciio showed that $22 / 28$ pts responded to autologous blood injection ${ }^{20}$. we used autologous plasma instead of blood to decrease the volume of injection and also to avoid the deposition of iron locally following breakdown of Rbcs .Mishra and pavelko introduced platelet rich plasma technique in the treatment of chronic elbow tendonitis. ${ }^{21}$ klein et al ${ }^{23}$ reported in their study that TGF $\beta$ increase type 1 collagen production in tendon sheath fibroblasts.these mechanism may be work in our study also.further study of autologous plasma versus whole blood or steroid should also be performed in the future.the data suggests autologous plasma may be an alternative to surgery in patients with this disorder of its kind.To conclude the use of autologous plasma injection method provides biologic healing and is an effective solution for refractory lateral epicondylitis of humerus and similar disorders.

\section{References}

[1]. Canale S.Terry,Beaty J.H,Campbells textbook of orthopaedics,pg 2634-38, $11^{\text {th }}$ edition

[2]. Leadbetter,W.B.cell matrix response,clin.sports medicine,11:533-578,1992.

[3]. Jozsa,L.G,Overuse injury in tendon,p164-253;1997.

[4]. Regan,W.Wold,L.E, Conrad,R;AND morry,a m sports med,20:746-49,1992.

[5]. Nirschl, r.P.: Tennis elbow tendinosis: pathoanatomy, nonsurgical and surgical management. In Reetitive Motion Disorders of the upper Extremity, pp. 467-479. Edited by S.L. Gordon, S.L.Blair,and L,J,fine. Rosemont, Lllinois, American Academy of Orthopaedic Surgeons, 1995

[6]. Clancy,W.G., Jr., Neidhart,D. and and Brand,R.L.: Achilles tendonitis in runners : areport of five cases. Am j. Sports Med.,4:46-57,1976.

[7]. Curwin,S.,and Stanish,W.D.: Tendinitis, Its Etiology and Treatment. Lexington, Massachusetts,Collamore Press, 1984.

[8]. Nirschl, R.P.: Patterns of failed tendon healing in tendon injury. In Sports-Induced Inflammation: Clinical and Basic Science Concepts, pp. 609-618. Edited by W.B. Leadbetter, J.A.Buckwalter, and S.L.Gordon.Park Ridge, Lllinois, American Academy of Orthopedic Surgeons, 1990

[9]. Stahl, S., and and kaufman, T.: the efficacy of an injection of steroids for medial epicondylitis. A prospective study of sixty elbows. J. Bone and Joint Surg., 79-A : 1648-1652, Nov. 1997.

[10]. Cyriax, J.H.: The pathology and treatment of tennis elbow. J.bone and Joint Surg., 18: 921-940,Oct. 1936.

[11]. Woo, S. L-Y., An, K.n, Arnoczky, S.P., Wayne, J.S., Fithian, D.C., and Myers, B.S.:Anatomy,Biology, and biomechanics of tendon, ligament, and meniscus. In Orthopaedic basic Science, pp. 45-87. Edited by S.R.Simon. Rosemont, Lllinois, American Academy of orthopaedic Surgeons, 1994.

[12]. Nirschl, R.P.: Elbow tendinosis/tennis elbow. Clin.Sports Med., 11 851-870,1992.

[13]. Amadio, P.C.: Tendon and ligament. In Wound Healing: Biomechanical and Clinical Aspected, pp. 384-395. Edited by I.K. Cohen, R.F.Diegelman, and W.J.Lindblad. Philadelphia,W.B.Saunders, 1992.

[14]. Smidt N, Assendelft W, Arola h, et al. Effectiveness of physiotherapy for lateral epicondylitis: a systematic review. Am Med. 2003, 35: $51-62$.

[15]. Altay T, Gunal I, Ozturk H. local injection treatment for lateral epicondylitis, Clin orthop Relat Res. 2002,398:127-130. Newcomer K, Laskowski E, Idank D, McLean R,Egan K

[16]. Corticosteroid injection in early treatment of lateral epicondylitis. Clin J Sports Med. 2001,11:214-222

[17]. Jobe F, Ciccotti M. Lateral and medical epicondylitis of the elbow. J Am Acad Orthopm Surg. 1994, 2: 1-8. 
[18]. Melikyan E, Shahin E,Miles J, Bainbridge L. Extracorporeal shock wave treatment for tennis elbow: a randomized double-blind study. J. Bone Joint Surg Br. 2003, 85: 852-855.

[19]. Einhorn T. clinical applications of recombinant human BMPs: early experience and future development. J Bone Joint Surg Am. 2003, 85(suppl 3): 82-88.

[20]. Edwards SG, Calandruccio JH.J Hand Surg Am. 2003 Mar, 28(2): 272-8.

[21]. Allan Mishra, MD, and terri Pavelko, PAC, PT the American Journal of Sports Medicine, Vol. X, No. X.

[22]. Young Lea moon, 1MD, Sueng-Hwan Jo,1MD, Chang Hun Song, 2,4Md, PhD, Geon Park, 3MD, phD, Geon Park, 3MD, phD, Hyun Ju Lee, 4 MS, Soo Jeong Jang, 4 MS Ann Acad med Singapore 2008, 37:559-63.

[23]. Klein M, Yalamanchi N, Pham H, Longaker m, Chang J. Flexor tendon healing in vitro: effects of TGF-b on tendon cell collagen production. J Hand Surg Am. 2002, 27: 615-620. 